\title{
Modelling Plant Variation Through Growth
}

Lisa Streit, Pavol Federl, Mario Costa Sousa

Department of Computer Science, University of Calgary

email: streitl|federl|mario@cpsc.ucalgary.ca

\section{Abstract}

This paper introduces a method for creating naturally varied plants from a given basic plant model. Previous techniques create variation in plants by introducing local randomness to the plant model's description. However, randomness is restricted by the model's parameterization and lack of correlation between local features making varying global properties (e.g. branch and stem curvature) difficult. We present a biologically-based method which mimics the underpinnings of variation in real plants. This method uses a feedback control system to simulate the biological growth mechanism by which a plant naturally responds to environmental factors. We show that our technique creates more realistically varied models by modelling growth responses to stimuli, and provides a method for quickly creating numerous similar models, none of which are exactly alike.

\section{Reference}

L. Streit, P. Federl, and M.C. Sousa: Modelling Plant Variation Through Growth. In Computer Graphics Forum 24 (3), pp. 497-506, 2005. 


\title{
Modelling Plant Variation Through Growth
}

\author{
L. Streit, P. Federl, M. C. Sousa ${ }^{\dagger}$ \\ Department of Computer Science, University of Calgary, Canada
}

\begin{abstract}
This paper introduces a method for creating naturally varied plants from a given basic plant model. Previous techniques create variation in plants by introducing local randomness to the plant model's description. However, randomness is restricted by the model's parameterization and lack of correlation between local features making varying global properties (e.g. branch and stem curvature) difficult. We present a biologically-based method which mimics the underpinnings of variation in real plants. This method uses a feedback control system to simulate the biological growth mechanism by which a plant naturally responds to environmental factors. We show that our technique creates more realistically varied models by modelling growth responses to stimuli, and provides a method for quickly creating numerous similar models, none of which are exactly alike.
\end{abstract}

Categories and Subject Descriptors (according to ACM CCS): I.3.7 [Computer Graphics]: Three-Dimensional Graphics and Realism

\section{Introduction}

Creating a natural-looking plant model is not a simple task; creating hundreds of plants for a scene is even more difficult. In the quest to attain realism, individual plant models have become increasingly complex through the addition of detail. Thus, manually modelling each individual plant in a scene is not acceptable. Our objective is to design an automatic, controllable approach for generating many naturally varied models from a single given model with little effort. This is achieved by subtly altering the overall shape of stems, branches and leaves requiring local natural variation to be correlated over the entire lengths of these elements.

Previous techniques to automatically generate collections of similar models include instancing [ $\mathrm{DHL}^{*}$ 98], interpolation and stochastic modelling [FF80]. While these techniques are sometimes useful there are weaknesses. With instancing the exact repetition of a small set of models may be immediately recognized. Interpolation between a small set to create intermediate models is dependent on both the interpolating function and the interpolated parameters. Often the results look too mechanical due to exact smooth interpolation. Stochastic modelling adds randomness to the model's description and can be combined with instancing and interpolation. However, variation is limited by the model's parameterization and the qualities of random noise. Since parameters typically represent local features, random alteration causes local variation which is globally uncorrelated. Control and predictability are lost, leaving no assurance of the model's resemblance to the desired biological object.

\footnotetext{
$\dagger\{$ streitl,federl,mario\}@cpsc.ucalgary.ca
}

To model shape variation of branches, stems and leaves local variation must be correlated to collectively define overall shape. We correlate variation by approximating the process of natural plant variation. In the world of living structures, growth defines form [Tho61]; thus, variations in the growth process create variations in form. We model variation by simulating growth responses. During growth a plant encounters an abundance of stimuli to which its type and degree of response result in variation across similar plants. Due to the stimuli's abundance and lesser importance to the response, it is infeasible to model every influence or stimuli thus an approximation to combined influences is used. These correlated responses which define the overall shape of branches, stems and leaves are the focus of this paper. By introducing the same "type" of variation found in real plants populations, without exceeding or deviating from it, the results gain realism while the variation is constrained to maintain a resemblance to the original model or object.

This paper shows that modelling plant structure variation through growth process simulation results in naturally varied plant models. First, we present an approach to vary shape by closely simulating differential growth through surface hormone changes. We then abstract this approach to alter the plant's skeleton by approximating plant growth responses and movements. We show that this method can create natural variation at multiple levels of detail and can quickly create a collection of similar plants with little effort.

\section{Background}

Creating a collection of different looking, yet similar objects is a common problem in natural phenomena modelling and 


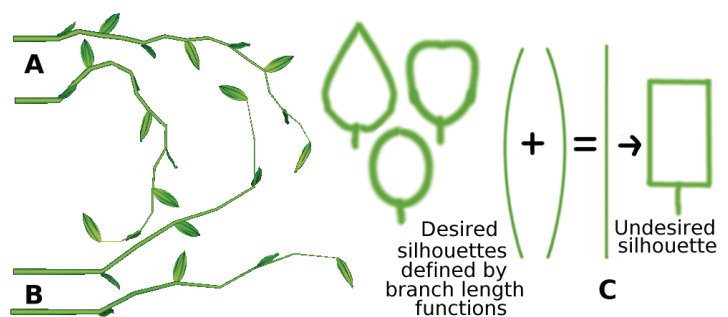

Figure 1: (A) and (B) Random stem segment angle variation in which overall shape is lost. (C) Interpolation of two desired silhouette curves defining branch length yield an undesirable straight silhouette.

can be paralleled with the study of natural plant variations. Just as Remphrey and Prusinkiewicz [RP97] categorize natural variation within a single plant structure as random or organized, modelling methods include random variation techniques which perturb a model's parameters randomly around a default value (i.e. stochastic modelling [FF80]) and organized techniques which methodically alter parameters according to predefined interpolants and functions as Prusinkiewicz et al. did with single plants [PMKL01]. While both random and organized methods produce sufficient results in some cases, both tend to lack predictability and control especially over global properties due to randomness or local feature specification and both can lack correct or complete ranges of variation faithful to botanical structures without careful specification of the parameters. These complications are shown in Figure 1.

Lintermann and Deussen [LD99] mention plant modelling techniques as having two separate motivations and goals: 1) to simulate natural plant development and is biologically motivated and 2) to generate only visually correct shapes of plants [DHL*98]. Our aim is the latter, but to achieve visually plausible results by approximating growth responses. Similar to Lintermann and Deussen our aim is to maintain user control and predictability of results, but in contrast, we automatically add variation instead of interactively creating varied plants. Weber and Penn [WP95] specify a model for creating plant geometry and model curves and stems as a functional specification through local segment alteration with random perturbation. So, while the local perturbations are globally correlated, exact functions directly control shape variation and thus require function alteration. Aono and Kunii [AK84] provide an interactive method of indirectly specifying variation using attractors and inhibitors to alter stem angles, similar to our approach. However, their method is not automatic nor biologically based and tends to have some of the affects of organized approaches.

Other methods of creating varied plants tend to be biologically motivated and simulate natural plant development. In an effort to preserve control some have developed customized procedural models [Opp86, $\mathrm{dREF}^{*} 88$, Hol94] that have incorporated overall shape through use of "functional modelling", free-form deformation and tropisms. Functional modelling as well as the rules defining tropisms have the same drawbacks of organized approaches mentioned above and free-form deformation is very user intensive. Some methods incorporate strict detailed [dREF*88] botanical rules which control local properties [PL90]. These methods add variation by functionally (random or otherwise) altering local parameters or by probabilistically selecting development rules (e.g. Stochastic L-systems), but have little control over global shape, such as shape of stems, branches and leaves. Rules defining responses to environmental factors (e.g. branching, space occupancy etc...) [Coh67, BM02, Gre89] have also been presented, but tend to have strong stochastic components and are less controllable or need multiple iterations to achieve a desired result. Others tend to define detailed biological local responses [PJM94] representing specific stimuli [MP96, COMM94] or complex physical simulation [HBM03].

Our aim is to automatically add believable variation to an existing satisfactory model regardless of creation approach or model geometry. Variation is added to the skeleton of stems, branches and leaves, without geometric or physical surface knowledge and is generated by approximating the natural growth process to ensure plausible results. Modelling variation requires modelling form, and the study of form has been ongoing for decades. D'Arcy Thompson [Tho61] recognized the relation of growth to biological form, stating that form in plants is determined by the rate of growth in various directions. Varying growth rates can be observed on multiple scales such as cell division, internode elongation, rate of bending of stems or roots, etc. Simulating growth to create form is thus a natural approach to generating variation at multiple levels of detail. The next two subsections overview plant growth motions and a relevant biological model.

\subsection{Plant Growth Movement and Form}

Plant growth occurs both at the apex or tip as well as in a specific region displaced from the tip. As the plant grows, this growth region migrates and differential growth combined with the displacement of the growth region cause the apex to "move" as the plant grows upward. This phenomena has been observed in both plant stems and roots [Han00]. These growth related movements can be directly related to a particular stimulus (tropic), or be triggered by the stimulus but not closely related (nastic) [SNSK08].

The two most commonly studied tropic movements are: 1) phototropism, tendency of growth toward (e.g. shoots) or away (e.g. roots) from light, and 2) gravitropism, tendency of growth toward (e.g. roots) [BBA89], away (e.g. shoots), or at some fixed angle to the direction of gravity [FD97]. Figure 2 shows sunflower stems undergoing a negative graviresponse due to pot rotation (from [Han00]). As shown, the pot of sunflower shoots was rotated and the shoots responded by bending upward. While a complete explanation for growth movements is active biological research, we use these as the basis for adding variation to plant models. The most common nastic response can be described as oscillatory movements during growth and can initiate tropic responses (e.g. climbing plants). There is ongoing debate over whether these movements are caused by an internal mechanism or the "over-shoot" of gravitropic responses [Joh97]. 


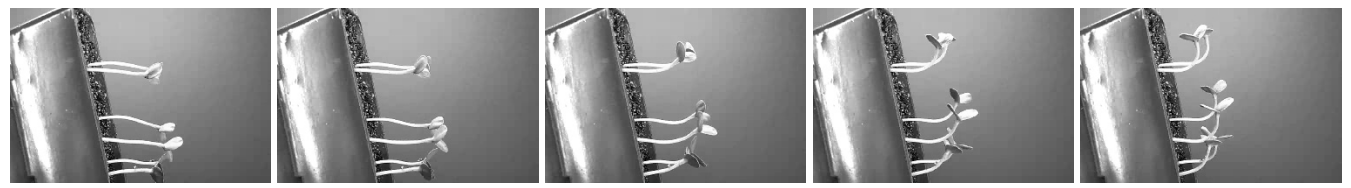

Animation frames from Roger Hangarter [Han00] with permission.

Figure 2: Within one hour of pot displacement the sunflower shoots responded negatively (away) to gravity. Frames at 0, 40, 60, 90 and 120 min. after displacement.

\subsection{Biological Models of Movement and Growth}

The majority of gravitropic response research has been with roots because of their quick response, relatively simple structure, and suitability for laboratory experimentation [BPB94]. Observations and models in this area are summarized here. Roots have a region of growth displaced from the root cap or tip (see Figure 3(c)). Darwin [Dar80] concluded that the root cap is necessary for gravitropic sensing and determination of the root's gravitropic response. Barlow et. al. [BBA89] concluded that growth rates (RELELs - relative elemental rates of elongation) may differ around the root within the growth region. They used RELELs to characterize the growth pattern over the organ, creating differential growth and curvature.

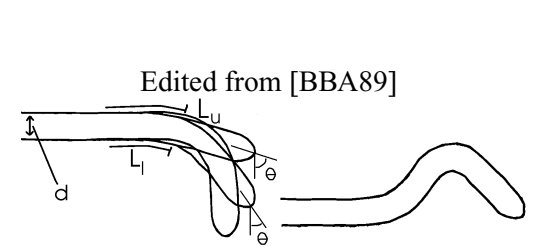

(a)

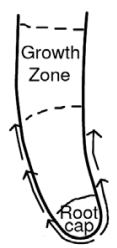

(c)
Figure 3: (a) Root tip bending. $\left(M_{u}, M_{l}\right)=(2.0,1.0),\left(\mu_{u}=\right.$ $\left.\mu_{l}\right)=3.0, \sigma_{u}=\sigma_{l}=1$, tip angles: $t=(0.126,0.380,0.633), \theta=$ $(18.1,54.5,90.8)$ (b) $\mu, \sigma$ alteration. $\left(M_{u}, M_{l}\right)=(3.0,1.0)$, $\left(\mu_{u}, \mu_{l}\right)=(3.0,5.0),\left(\sigma_{u}, \sigma_{l}\right)=(0.7,1.0)$ (c) Illustration of root and substance transport.

Barlow et al. [BBA89, ZBB97] model a plant root as a constant-diameter $d$ cylinder. They simulate a $2 \mathrm{D}$ longitudinal slice of the root, constraining bending within the plane. A RELEL is assumed for each point $s$ at time $t$ on the upper $L_{u}$ and lower $L_{l}$ flanks of the slice and is associated with an angle of the tip to the vertical (displacement) $\theta$ at some earlier time $t^{\prime}$. The time-lapse indicates lag between the sensed displacement and the resulting response. Differential growth results from asymmetric RELELs. They used a Gaussian distribution of RELELs $=\operatorname{Mexp}\left(-(x-\mu)^{2} / 2 \sigma^{2}\right.$ where $x$ is the distance from $s$ to the tip, $\mu$ is the value of $x$ at the maximum $M$ RELEL, and $\sigma$ is the distance from $\mu$ where $R E L E L=0.607 M$. Differences in parameters cause growth rate differences around the root at a given time.

The tip angle $\theta$ at any time is determined by the lengths of $L_{u}$ and $L_{l}$. Varying $M$ while keeping $\sigma$ and $\mu$ the same for $L_{u}$ and $L_{l}$ alters the growth rate and flank length proportional to $M$ with proportionality constant $\alpha$ as follows:

$$
\theta=\frac{L_{u}-L_{l}}{d}=\frac{\alpha\left(M_{u}-M_{l}\right)}{d}
$$

This achieves bending over time (see Figure 3a). Varying $\sigma$ independently on $L_{u}$ and $L_{l}$ results in curves of different radii, and altering $\mu$ over time can form kinked shapes similar to corn roots [BBA89, p.79] (see Figure 3b).

Barlow et al. [BBA89, p.79] suggest achieving more elaborate curves through complex rearrangements of growth rates (e.g. $\theta$ a function of other parameters like $\mu$ or asymmetric RELEL distributions) or interactions of different tropisms. Barlow et al. [BPB94] note two more results of interest to our approach. First, the rate of bending is constant independent of $\theta$. Second, the relation of the lag (between sensor and response) to the response, can cause bending to overshoot the target re-initiating an gravitropic response on the other side to simulate the commencement of nutation. The relation of the tip angle to overall shape and the observation of the relation between lag and response forms the basis of the structural approach described in Section 4.

\section{Surface-based Variation}

Our first approach to modelling variations simulates alterations in surface growth rate of a root according to the uneven distribution of a plant hormone called auxin. The auxin concentration inhibits growth leading to bending. The impact of differences in growth rate on resulting root shape is comparable with how the distribution of Barlow's RELELs [BBA89] affects the resulting tip angle of a root. This biological simulation is used to verify the results of our skeletal based method presented in Section 4 .

The assumptions we used to construct our model were taken from [MTR* $\left.{ }^{*} 4\right]$, and are briefly outlined below. Auxin is transported through the root's center toward the tip at some constant rate. The auxin is then distributed at the tip by a gravitropic mechanism, as a function of orientation of the tip with respect to the gravity vector (angle $\theta$ see Figure $3 a$ ). A larger fraction of the available auxin is redirected to the side of the root tip that is oriented toward gravity. When the direction of the tip and gravity match, auxin is equally distributed. Once the auxin is distributed at the tip, it is transported by a polar transport mechanism along the surface of the root back toward the plant, as illustrated in Figure 3c. Auxin modulates growth rates within the growth zone by inhibition. Uneven auxin concentrations cause uneven (differential) growth and consequent bending, a behavior consistent with Barlow et al. models [BBA89, ZBB97]. Bending continues until the tip is realigned with gravity and an even distribution of auxin is restored.

Based on the above assumptions, we constructed the follow- 

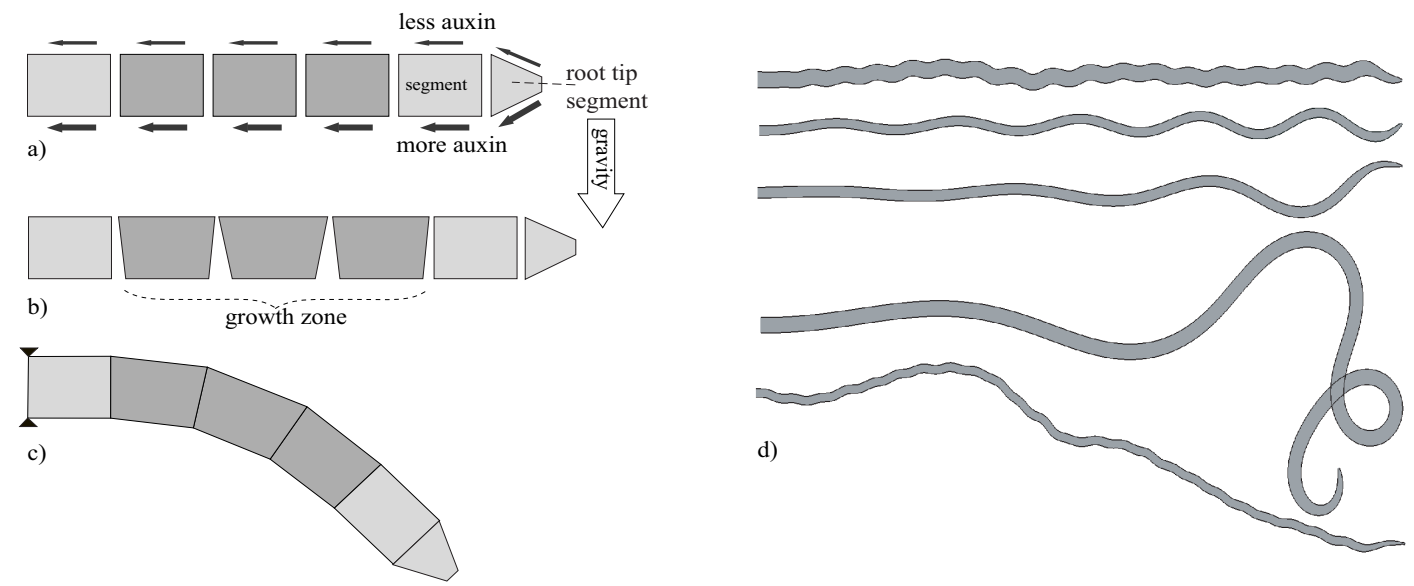

Figure 4: (a-c) Simulation model of a gravitropic response in a developing root. (d) Various root shapes produced by the model.

ing two-dimensional simulation. We first discretize the root into multiple segments and a single root tip segment, as illustrated in Figure 4a. Each segment is associated with two types of information, the level of auxin at its top and bottom sides, and the segment's shape. The segments are initially rectangular, although they deform throughout the simulation. The core of the simulation consists of the main loop, where the development of the root is incrementally simulated in time steps of $\Delta t$.

Each iteration through the main loop consists of three steps. First, we calculate the concentrations changes of auxin in each segment in time $\Delta t$. This amounts to simulating polar transport oriented in the direction away from the root tip and accounting for addition of new auxin at the root tip from the root center and re-distributing it by the gravity sensing mechanism.

In the second step, after the new values of auxin in each segment are calculated, we simulate the growth of each segment. The shape of each segment is changed by elongating their top and bottom sides according to growth rate, similar to RELELs [BBA89]. The growth rate of a particular side of a segment is a function of two variables: the distance from the root tip, and the concentration of auxin at the side of the segment. The distance from the root tip determines the location of the growth zone [BBA89]. The root grows faster near the center of the growth zone and tapers off away from the center. This preliminary rate is then modified according to the level of auxin associated with the side of the segment. The resulting growth rate is then applied to calculate the new lengths of the sides of each segment and update the segment shape, resulting in rhomboidal-shaped segments (Figure 4b). To prevent segments from becoming too large, they are dynamically subdivided upon reaching a threshold size.

In the final step of the simulation loop, after the individual segment shapes have been computed, we assemble the resulting root shape as illustrated in Figure 4c. This is accomplished by first fixing the last segment of the root to some predefined location. The next adjacent segment is then translated and rotated so that its left side matches the last segment's right side. This process is repeated iteratively until the shape of the whole root is reconstructed. The root bending is thus an emergent phenomenon in our simulations. Various root shapes obtained using our 2D simulation are shown in Figure 4d. These results were generated by varying some of the simulation parameters, such as the size and location of the growth zone, the auxin transport rate, the inhibition effect of auxin on growth, etc.

We also extended our gravitropic simulation of roots to three dimensions. We assume that the initial shape of the young root is cone-like, which we discretize into cylindrical segments of equal heights. Each such segment's surface is then discretized into a polygonal mesh. As the auxin is transported through the segments' surfaces, the mesh polygons undergo growth. The result of uneven auxin distributions is non-uniform growth, which manifests itself by transforming the initial cylinder-like segments into more wedge-like shapes. By iteratively assembling the segments, similar to the process described for the $2 \mathrm{D}$ model, the $3 \mathrm{D}$ shape of the root can be reconstructed.

\section{Skeletal-based Variation}

We model plant variations through changes in the structure or skeleton by abstracting the surface growth responses shown in Section 3, and relating them to the plant skeleton and changes in tip angle similar to Barlow's work (see Section 2.2). Starting from a base model skeletal representation, variations are added by simulating growth responses to influences or stimuli (see Section 2). The skeleton is subdivided into growth steps and at each step we monitor and respond to the difference between the desired (base model) and current growth directions. This mechanism is modelled using a control system.

We use a base model's skeleton to define the desired direction or natural course of growth. Then by subjecting this skeleton to the same stimuli as real plants and compensating for the surface offset from the skeleton, we apply adjustments directly to the skeletal representation. Once the modified skeleton is obtained, the surface is recreated and a new 
varied model which resembles the base model is generated as shown in Figure 5.

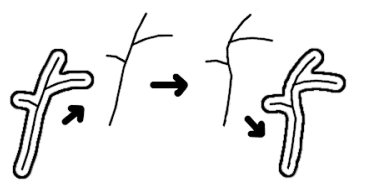

Figure 5: Variation of an initial "skinned" skeleton occurs by altering the skeleton and "re-skinning".
Using a base model for determining the desired course of growth both parallels what may naturally occur and has benefits to modelling. In nature, plants of a particular type typically have a common general direction of growth. We assume this general direction is the desired course of growth and the stimuli or influences trigger responses resulting in variation. Simulating the stimuli/response mechanism to maintain a desired course of growth for plant modelling has a dual purpose: realism and control. Simulating biological plant responses aid in creating more realistic variations. Defining the desired course of growth from a base model ensures a resemblance to the base model.

A closed-loop feedback control system is used to simulate the response mechanism. The control system has a sensor, a response and a lag between these components which all parallel the growth mechanism described in Section 2.2. This mechanism is used to maintain a resemblance to the base model skeleton. The control factor is the relation of the growth rate to the tip angle or displacement between the current and desired course of growth similar to Barlow (see Section 2.2). This displacement angle is computed at regular intervals and then depending on the growth rate, lag and external influences, an adjustment to the current growth direction is made to compensate for the influences.

\subsection{Closed-loop Feedback Control Systems}

Control systems [FPEN94] typically have an input, a sensor which determines error, and a response mechanism which compensates for error. Errors arise as differences $e$, between the input data $r$ and the sensed data, in the sensor $v$ or from an external disturbance $w$. The sensor measures differences $e$ between the input and the current sensed data, which in our case are the desired and current growth directions respectively. Figure 6 shows a typical closed-loop feedback control system. $D(s)$ is the transfer function to convert input $(r$ and $e$ ) to computed adjustment $u$ at time $s . G(s)$ is the transfer function that takes the adjustment, $u$ and (possibly adds a disturbance $-w$ ) and generates a response. The sensor then remeasures and computes the error, $e$ and possibly introduces error, $v$.

The transfer function $D(s)$ computes an adjustment proportional to the amount of error in the system at a given time. This means the computed adjustment to the tip angle is proportional to the displacement (angle) of the tip from the predetermined course of growth. So, $D(s)=\kappa \theta$, where $\theta$ is the difference between the current growth direction and predetermined growth direction (see section 4.2) and $\kappa$ is the proportionality coefficient. While there are many types of controllers [FPEN94] for reducing disturbances (influences) in a system, we found using this proportional control is sufficient

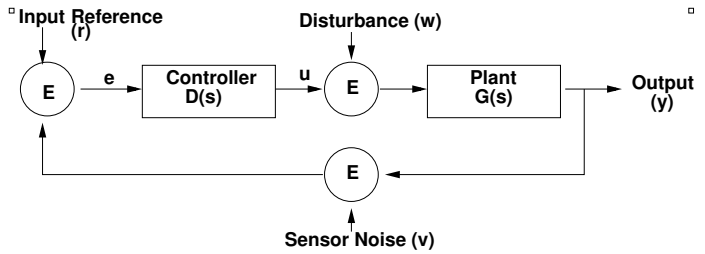

Figure 6: In a typical closed-loop feedback system the controller $D(s)$ transfers input ( $r$ and e) to an adjustment $u$. $G(s)$ responds to $u$ (and disturbance, $w$ ). The sensor then recomputes error, e and may introduce error, $v$.

in most cases. Other types of controllers may be required when adjustments for change in error (derivative) or accumulation of error (integral) are needed (see Section 4.2.1).

\subsection{Modelling Growth Responses to Influences}

We model natural growth responses by assuming continuous growth and using a control system to simulate plant responses. The input $r$ to our control system is the skeleton of the base model as described by an L-system. The sensor $v$ and external disturbance $w$ errors represent stimuli or influences the plant responds to. The transfer function's ability to compensate for lag and error determine if the system is stable (i.e. error is bounded), convergent or divergent. Furthermore, our assumption of growth for movement means that compensation for influences is dependent on growth and ultimately on the ratio of growth rate to error rate. Movements which do not require growth can possibly be incorporated into the model later, when more is known about their impact.

\subsubsection{Modelling Responses}

The growth process is simulated by dividing the plant development into discrete growth steps. At each step the plant tip senses how far displaced (in degrees) the tip is from the desired direction of growth. Once this difference is measured, the plant then determines how to alter growth rates to induce a change in the orientation of the apex. This is simulated by the controller computing a change in growth direction using the transfer function $D(s)$. A signal is then passed along the stem where after some lag it arrives at the growth zone. This is simulated by creating a signal at each growth step with a lifetime and a corresponding adjustment or change in growth direction. Once the signal expires, the response is activated altering the skeleton reflecting the altered growth rates.

Figure 7 shows growth simulation by first subdividing the segment into discrete growth steps. At each step the growth direction difference is sensed, $\theta$ and an adjustment computed. A signal $S n$ for the adjustment is generated with a lifetime $(m)$ which decays and is transmitted to the growth zone. As growth proceeds, the prior signals decay and new signals are generated. In this example each signal has a lifetime of two and the growth rate, signal transmission rate are equal, and decay rate is constant. Thus, the plant grows upward as fast as the signals travel downward and decay. Once the first signal expires (at the far right) the skeleton is adjusted. For simplicity, adjustment is the full difference (i.e. $\kappa=1$ ). With a constant growth rate the response always occurs the same 


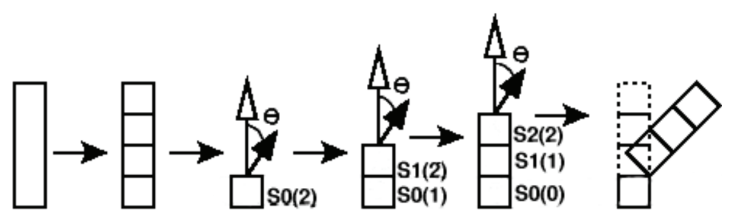

Figure 7: At each step the growth direction difference, $\theta$ is sensed and an adjustment computed. A signal Sn carries the adjustment which is activated when the signal "expires".

distance from tip. By altering the growth rate, transmission rate, lifetime (i.e how long a signal lasts with constant decay rate), or decay rate of various signals, the growth region may migrate causing signals to convolve, requiring integral or derivative control (see Section 4.1). In the examples reported in this paper, growth occurs in the growth zone (near the tip) only, however in plants growth also occurs as elongation of stems. This may be simulated by similarly elongating each subdivided growth step and adjusting the signal decay appropriately.

\subsubsection{Modelling Stimuli or Influences}

There are two types of possible influences that cause variation: imperfect sensing and external or environmental stimuli. The plant senses how far (angle) the apex is from the ideal predetermined direction or path of growth by sensing both the current growth direction $H_{\text {curr }}$ and the desired or target growth direction $H_{t a r}$. In reality there are numerous factors (e.g. hydration, growth rate etc.) that may cause imprecision in this measure. Due to imperfect sensing, $H_{\text {curr }}$ may not accurately represent the current growth direction. In our model the sensor error is approximated by random radial Gaussian distribution about $H_{\text {curr }}$ as shown at left, creating the sensed growth direction $H_{\text {sense. }}$. The difference in growth directions is then determined by computing the angular difference between two normalized vectors, $H_{\text {sense }}$ and $H_{\text {tar }}$ as: $\operatorname{acos}\left(H_{\text {sense }} \cdot H_{\text {tar }}\right)$. The controller uses this difference to compute an adjustment (see Section 4.1).

While adjustment computation is exact using the defined transfer function and the sensed difference, the plant may react without knowledge of external stimuli. The external stimuli represent environmental factors that may affect the growth direction aside from those which define the predetermined course of growth. Since these factors (e.g. wind, growth proximity etc.) are so numerous we model them in two ways: randomly and functionally. The random model represents the statistical averaging of numerous seemingly random factors resulting in a random influence with a Gaussian or uniform distribution. Alternatively, to explore how a specific, possibly dominant, stimulus affects the growth direction, a function can be specified which represents this stimulus or overall target growth direction. Figure 8 shows two examples of user specified target (left bottom) and disturbance functions (left top). These functions are applied with the target function in one orthogonal plane (middle) to define overall shape and the disturbance in a second orthogonal plane to show local variation (right). A more integrated

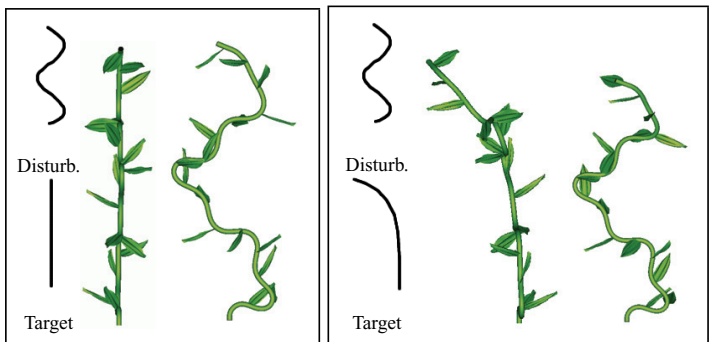

Figure 8: Two examples of user specified functions (left) applied orthogonally (right).

example of the use of target function specification is shown in Figure 13 and is detailed in Section 5.

The difference between imperfect sensing and external stimuli is, the former is directly compensated for by the controller, while external stimuli are only compensated at the next stage of sensing and correction (see Figure 6). Consequently, imperfect sensing impacts variation less.

\subsection{The Process and Form}

In addition to growth and error rate affecting form, the lag between the determination and activation of a response, as noted by Barlow et. al [BPB94] also affects form. In real plants the relation between lag and growth rate is not clear. In our model it is a ratio between the lag and the ability to adjust (i.e. size of adjustment $\kappa$ see Section 4.1) that impacts form the most. This ratio represents the plant response to stimuli and will be called the compensation ratio. The magnitude of the lag reflects the rate of response. The magnitude of the adjustment, determined by transfer function $D(s)$, reflects the number of adjustments required to compensate for an influence. No adjustment can be made without a response first signaling an adjustment, coupling these parameters into the compensation ratio. To provide an intuition and relation to the feedback control mechanism with a single initial disturbance, error $E(n)$ (i.e. $\theta$ ) at growth stage $n$, obeys the recurrence relation: $E(n)=E(n-1)-\kappa E(n-1-l a g)$, where $D(s)=\kappa \theta$. The compensation ratio, $(\kappa * \text { lag })^{-1} \approx 0.7$ has been experimentally determined to represent a stable system and is discussed below.

Consider a single initial influence on a plant, whose desired course of growth is straight upward (either toward a point light or away from gravity). The single influence simulates a change relative to gravity direction by displacing the plant $90^{\circ}$ shortly after growth is commenced, similar to the sun-
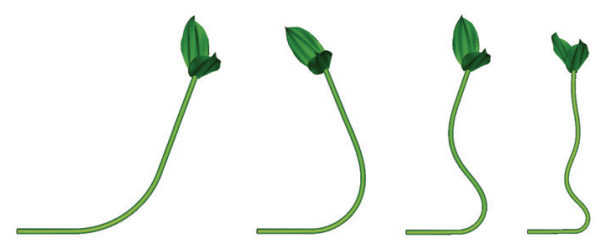

Figure 9: Varying lag and adjustment ability. At 20\% of shown growth a $90^{\circ}$ rotation occurred. Compensation ratio $=0.72$, lag decreasing left to right. Compare with Figure 2. 
flower animation in Figure 2. Figure 9 shows our model of a plant reacting to being turned $90^{\circ}$ at $20 \%$ of total shown growth. Varying amounts of lag and adjustment ability are used, however, all compensation ratios are fixed at 0.72 . As the lag magnitude decreases from left to right, the plant adjusts to the stimuli more quickly. Note the small amount of oscillation before convergence to the pre-determined course in the two rightmost plants due to the significant "overshoot" of the target.

The two parameters for controlling variation given a base skeleton, are the lag and the adjustment ability $\kappa$, which together form the compensation ratio. As shown in Figure 9 maintaining a constant compensation ratio and altering lag magnitude (i.e. altering $\kappa$ proportionally to lag) introduces variation at different levels of detail. The plant on the far right has variation of a much higher frequency than the plant on the far left. The overall plant shape is controlled using the base skeleton model and as shown all plants achieve a general upward course of growth despite being turned $90^{\circ}$. Altering the compensation ratio (i.e. changing lag disproportionally to $\kappa$ ) can alter the change in variation over growth as shown below. A compensation ratio from 0.5 to 1.2 effectively alters variation. The sensitivity of variation to lag magnitude depends on the number of subdivided growth steps used. All examples in this paper had lag $\in[4 \%-30 \%]$ of the number of subdivided growth steps.

As the compensation ratio is altered, the plant either compensates equally for the stimulus, under-compensates or overcompensates. If the plant equally compensates for the stimulus given the lag, then regular oscillation may occur. The lag is long enough, relative to the adjustment and stimuli magnitude, such that the adjustments regularly overshoot the target by a constant amount causing oscillation. However, if the plant under-compensates or overcompensates either a divergent or convergent form results, meaning that the plant either overshot the target by a increasing or decreasing amount respectively as shown in Figure 10. The plants were rotated $20^{\circ}$ at the start of growth. The group of stalks on the left have shorter lag than those on the right. Within each group the compensation ratio is increased from left to right to exhibit under-compensation, equal and overcompensation of the stimuli, respectively.
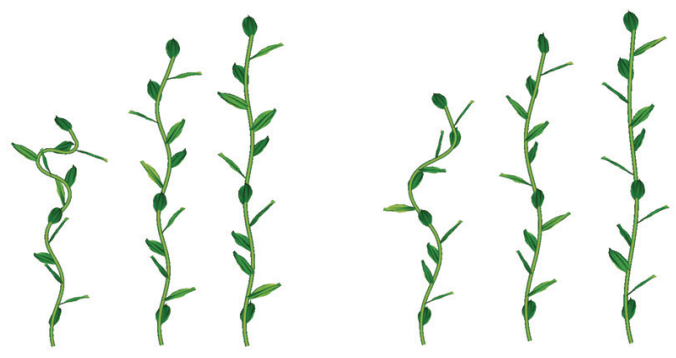

Figure 10: Two groups of differing lag and adjustment abilities. Left: smaller lag Right: larger lag. In each group the adjustment ability is under-compensated (left), equal (center) and over-compensated (right).

\subsection{Implementation and L-system Details}

The skeleton is sequentially varied proceeding from the root to the tip by adding variation to each subdivided skeletal segment (see Section 4.2.1). Assuming the existence of a method STRAIGHSKEL() for creating a straight skeletal segment of length $l$, we devised an algorithm to create a varied skeletal segment of the same length as shown below.

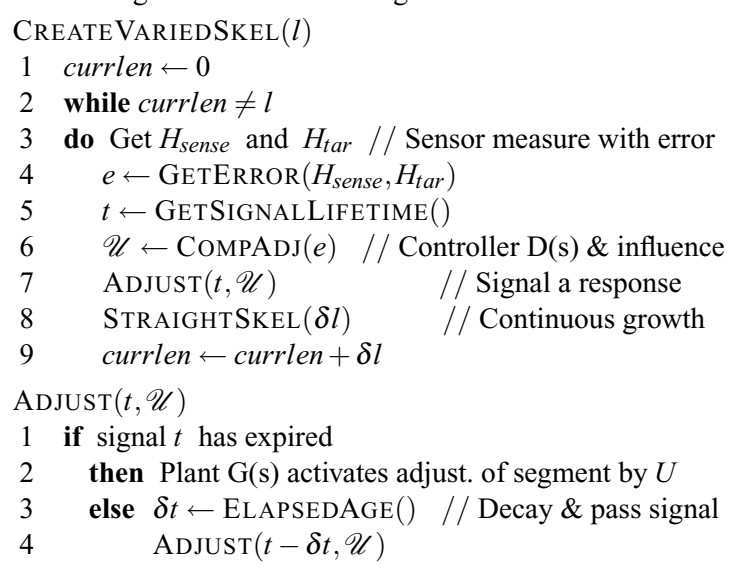

In our algorithm, small segments $\delta l$ are altered in orientation to create variation over length $l$. Note that segment addition or growth is continuous and an adjustment is made each time a subsegment $\delta l$ is added. However, the growth rate can be varied by altering $\delta l$ over time. In relation to the control system in Figure 6, at each growth step the sensor detects the current and target growth directions (line 3 ) and measures the error (line 4). The controller then computes an adjustment in orientation (line 6 ) and creates a response signal with lifetime $t$ (lines 5 and 7). This process is repeated until the length $l$ is achieved.

While our algorithm is general enough to introduce variation to any skeletal model defining $H_{t a r}$, we have chosen to use an L-system as the base model's skeleton and consequently implement the algorithm as an L-system. The details needed to convert most L-system plant descriptions to one which introduces variation is given in the appendix.

\section{Results}

Once the L-system is modified, the varied skeleton is generated in seconds on a $800 \mathrm{MHz}$ PIII with a GeForce FX graphics card. The development of the variation is, of course, directly dependent on the number of subsegments used. Figure 11 shows the base Lily model (left) and a varied model (right). This model has a compensation ratio of $\approx 0.7$ and large lag shown by the stem, branch and leaf curvature.

Figure 12 shows a model of Horneophyton with constant compensation ratio and differing amounts of lag and influences. An illustration and base model (L-system) are shown in (a) and (b). A constant compensation ratio is maintained, but altering lag results in variation of different frequencies (compare group $d$ with e) and altering the magnitude of the influence or stimuli alters the magnitude of the variation (compare within group $\mathrm{d}$ or e). A model with stochastic branch angle variation by $\pm 17.5^{\circ}$ degrees is shown in 

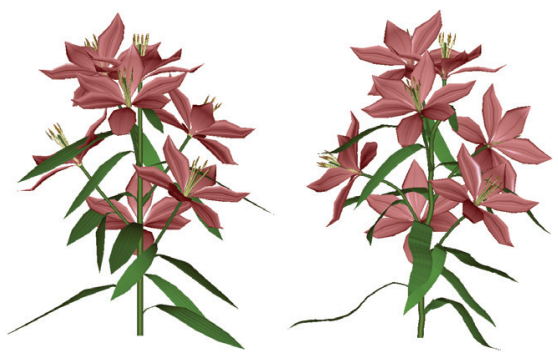

Figure 11: Right: Variations of Lily model at Left

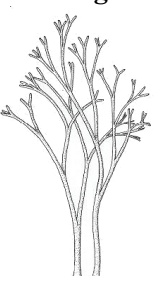

(a)
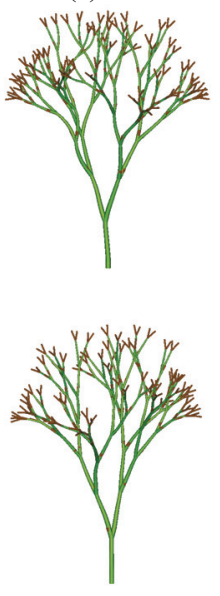

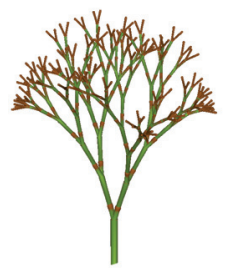

(b)

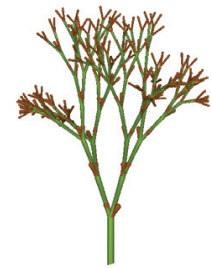

(c)
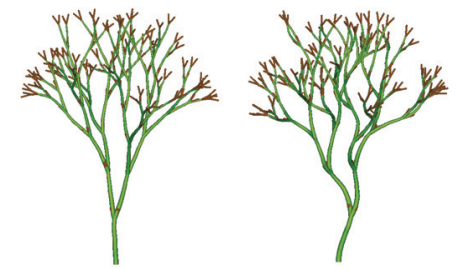

(d) small lag
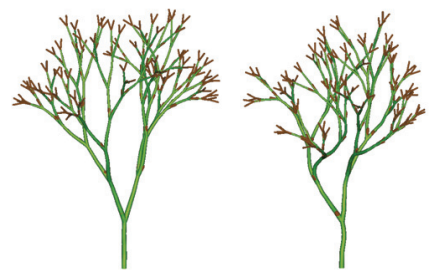

(e) large lag

Figure 12: (a) Illustration modified from Eggert (1974) (b) original model (c) random branch angles by $\pm 17.5^{\circ}$ maximum. (d) \& (e) slight overcompensation with increasing random external disturbance to right (d) small lag (e) large lag

(c). Note how the models in group (d) and (e) preserve the branch shape shown in (a) better than in (c).

Figure 13 shows alteration of the compensation ratio. A base plant model is shown on the left with three varied models on the right. The compensation ratio increases from left to right and thus the models show less divergence from the base model and lower frequency variation to the right. This model stochastically determines the location and number of branches, hence the differences in this regard. Finally, a dominate phototropic influence causes change in the desired growth direction along each branch from initially outward to upward. The desired growth direction is a weighting (similar to [AK84, PL90]) between an initial outward direction and upward influence using the functional specification described in Section 4.2.2. The result is all branches in all models generally bend upward, but due to other influences and lag in the adjustment each branch is slightly different.
Figure 14 shows two examples of our method used to quickly generate a collection of similar naturally varied palm trees. One base model of a palm tree was used as shown on the top, a real photograph on the left and the simulated models on the right. All plant models in each scene have the same variation parameters for simplicity. Note the correlated variation along the entire length of the stems. Once the base model and the location and scale of the plants were determined, these scenes were generated in minutes. The three palm trees in the center of the bottom images were purposely tilted by changing the desired growth direction.

By altering lag, compensation ratio and influences this method creates a range of frequency and magnitude variations of a base model comparable to natural plant variations. This technique is shown to be useful in creating natural collections of similar models quickly and effortlessly.

\section{Conclusions}

We showed that by coarsely simulating the growth response of plants to stimuli, we are able to approximate natural plant variation. Alterations were applied directly to the skeletal representation of a base model. A control system was used to closely simulate the natural growth process which ensured natural looking variation of a base model. The amount of variation and level of detail were controlled by maintaining a resemblance to the desired base model. Our technique correlates local variation with global shape to create variation along entire lengths of stems, branches and leaves. This notion of control and representation of natural variation cannot be achieved with previous instancing and stochastic techniques. While our method does involve the use of randomness to approximate the combined affect of many influences, the random function does not directly define the resulting variation in form. It is rather the simulated growth response to these random influences which define the resulting form. We showed that our technique can be used to quickly and efficiently generate numerous models of the same kind of plant in which each plant looks similar, but is not the same. As shown these varied models can be arranged to create a "natural-looking" collection of similar plants.

\section{Acknowledgments}

We would like to thank Dr. Prusinkiewicz for research discussions and paper comments and the Jungle Group at UofC particularly Brendan, Colin and Richard and the anonymous reviewers for their valuable comments. We also thank the late Alain Fournier, Roger Hangarter and Bill Reeves for use of their work. Thank you Wes, the Streit family, the Sahneys, Roger, Rob and JP for support and encouragement. This research was supported by grants from NSERC of Canada.

\section{References}

[AK84] AONO M., KUnII T. L.: Botanical tree image generation. IEEE Computer Graphics and Applications 4, 5 tion. IEEE Computer Graphic.
(May 1984), 10-29, 32-34.

[BBA89] BARlow P. W., BRAin P., AdAm J. S.: DifferenBARLOW P. W., BRAIN P., ADAM J. S.: Differen-
tial growth and plant tropisms: A study assisted by computer simulation. Environmental and Experimental Botany 29, 1 (1989), 71-83. 


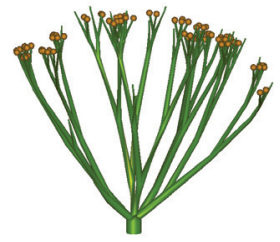

original

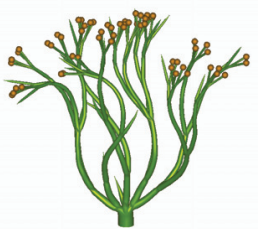

slight under compensation

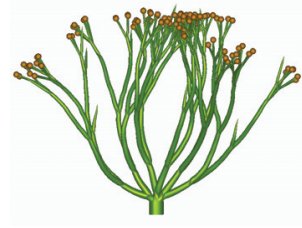

equal compensation

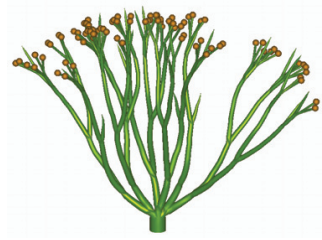

slight over compensation

Figure 13: Cooksonia Left: Original Model to Right: increasing compensation ratio.

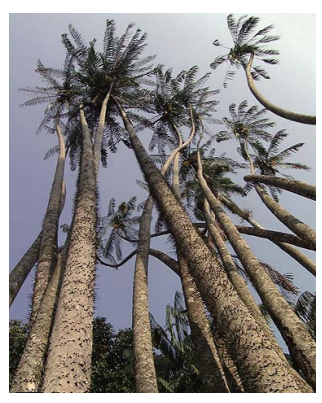

Photograph

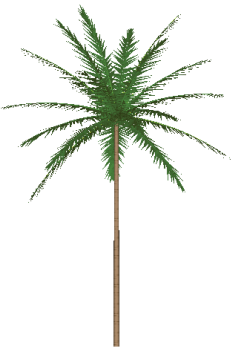

Base Model

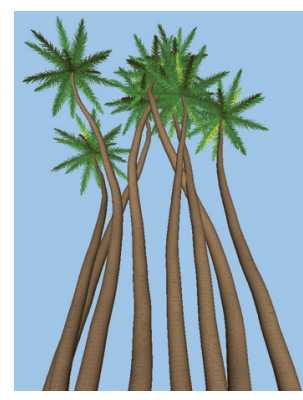

Simulated cluster

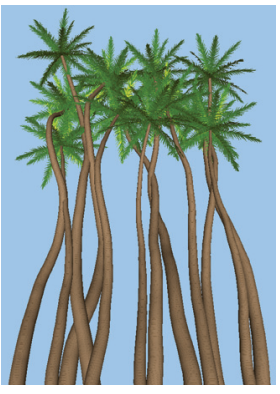

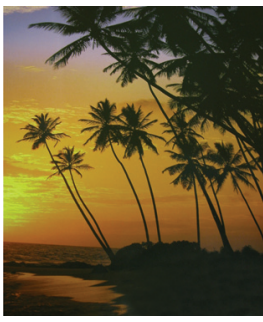

Photograph

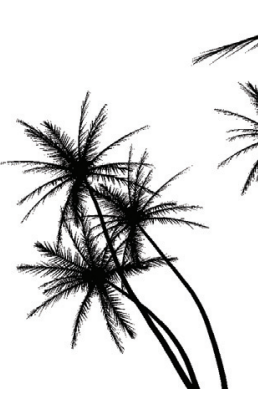

Figure 14: Top: Palm tree cluster Bottom: Palm trees on a beach. Right: background courtesy of Fournier and Reeves [FR86].

[BM02] Beneš B., Millán E.: Virtual Climbing Plants Competing for Space. In IEEE Proc. of Computer Animation (2002), Magnenat-Thalmann N., (Ed.), IEEE Computer Society, pp. 33-42.

[BPB94] BARLOW P. W., PARKer J. S., BRAIN P.: Oscillation of axial plant organs. Adv. Space Res. 14, 8 (1994), 149-158.

[Coh67] COHEN D.: Computer simulation of biological pattern generation processes. Nature, 216 (1967), 246-248.

[COMM94] Chiba N., OKawa S., Muroaka K., Muira M.: Visual Simulation of Botanical Trees Based on Virtual Heliotropis $m$ and Dormancy Break. Journal of Visualization and Computer Animation 5 (1994), 3-15.

[Dar80] DARWIN C.: The Power of Movement in Plants. J. Murray, London,UK, 1880.

[DHL*98] Deussen O., Hanrahan P., Lintermann B., MÉCH R., PHARR M., PRUSINKIEWICZ P.: Realistic modeling and rendering of plant ecosystems. In $S I G-$ GRAPH '98: Proceedings of the 25th annual conference on Computer graphics and interactive techniques (1998), ACM Press, pp. 275-286.

[dREF*88] de RefFye P., Edelin C., Francon J., Jaeger M., PUECH C. Plant models faithful to botanical structure and developmentr. In SIGGRAPH '88: Proceedings of the 15th annual conference on Computer graphics and interactive techniques (New York, NY, USA, 1988), ACM Press, pp. 151-158.
[FD97] FIRN R. D., DigBY J.: Solving the puzzle of gravitropism - has a lost peice been found? Planta 203 (1997), S159-S163.

[FF80] Fournier A., Fussell D.: Stochastic modeling in computer graphics. SIGGRAPH Comput. Graph. 14, SI (1980), 1-8.

[FPEN94] Franklin G. F., Powell J. D., Emani-Naeini A.: Feedback Control of Dynamic Systems. Addison Aesley Publishing Co. Inc., 1994.

[FR86] Fournier A., REEVEs W. T.: A simple model of ocean waves. In SIGGRAPH '86: Proceedings of the 13 th annual conference on Computer graphics and interactive techniques (1986), ACM Press, pp. 75-84.

[Gre89] GREENE N.: Voxel space automata: modeling with stochastic growth processes in voxel space. In $S I G$ GRAPH '89: Proceedings of the 16th annual conference on $C$ omputer graphics and interactive techniques (1989), ACM Press, pp. 175-184.

[Han00] HANGARTER R. P.: Plants in motion. http://sunflower.bio.indiana.edu/ rhangart/plantmotion/movements/ tropism/tropisms.html, 2000.

[HBM03] Hart J. C., Baker B., Michaelraj J.: Structural Simulation of Tree Growth . The Visual Computer 19(2-3) (2003), 151-163. 
[Hol94] Holton M.: Strands, gravity, and botanical tree imagery. Comput. Graph. Forum 13, 1 (1994), 57-67.

[Joh97] Johnsson A.: Circumnutations: results from recent experiments on earth and in space. Planta 203 (1997), S147-S158.

[KL02] KARWOWSKI R., LANE B.: Lpfg user's manual. http://algorithmicbotany.org/ ual. http://algorithmicbotany.org/ lstudio/LPFGman.pdf, 2002

[LD99] Lintermann B., Deussen O.: Interactive modeling of plants. IEEE Comput. Graph. Appl. 19, 1 (1999), 56-65.

[MP96] MĔCH R., Prusinkiewicz P.: Visual models of plants interacting with their environment. In Proceedings of the 23rd annual conference on Computer graphics and interactive techniques (1996), ACM graphics and interact
Press, pp. 397-410.

[MTR*04] Molendijk A. J., Tietz O., Ruperti B., PaPONOV I. A., PALME K.: Mechanisms of cell polarity establishment and polar auxin transport. In Polarity in Plants, Lindsey K., (Ed.). Ed.Blackwell publishing, CRC Press, 2004, pp. 192-240.

[Opp86] OPPENHEIMER P. E. Real time design and animation of fractal plants and trees. In SIGGRAPH '86: Proceedings of the 13th annual conference on Computer graphics and interactive techniques (New York, NY, USA, 1986), ACM Press, pp. 55-64.

[PJM94] PRUSINKIEWICZ P., JAMES M., M\&\#283;CH R.: Synthetic topiary. In SIGGRAPH' '94: Proceedings of the 21st annual conference on Computer graphics and interactive techniques (New York, NY, USA, 1994),
in ACM Press, pp. 351-358.

[PL90] Prusinkiewicz P., Lindenmayer A.: The Algorithmic Beauty of Plants. Springer-Verlag, 1990.

[PMKL01] PRUSinkiewicz P., MÜndermanN L., KaRWOWSKI R., LANE B.: The use of positional information in the modeling of plants. In Proceedings of $A C M$ SIGGRAPH 2001 (Aug. 2001), Computer Graphics Proceedings, Annual Conference Series, pp. 289-300.

[RP97] Remphrey W. R., Prusinkiewicz P.: Quantification and modelling of tree architecture. In Plant to Ecosystems, Michalewicz M. T., (Ed.). CSIRO Australia, 1997, pp. 45-52.

[SNSK08] Strasburger E., Noll F., Schenck H., KarsTEU G.: A text-book of Botany. Macmillan and Co. Ltd., London, 1908.

[Tho61] Thompson D. W.: On Growth and Form. Cambridge at the University Press, New York, 1961.

[WP95] WEBER J., PENN J.: Creation and rendering of realistic trees. In SIGGRAPH '95: Proceedings of the 22nd annual conference on Computer graphics and interactive techniques (New York, NY, USA, 1995), ACM Press, pp. 119-128.

[ZBB97] Zieschang H. E., Brain P., Barlow P.: Modelling of root growth and bending in two dimensions. Journal of Theoretical Biology 184 (1997), 237-246.

Appendix A: L-system Implementation Details

The base skeleton generally defines the desired course of growth and is described by a base plant's L-system. The L-system is a set of rules which define the plants construction [PL90]. Implementing our algorithm as part of an Lsystem requires a few details. The variation is added as the L-system is evaluated requiring two events at each evaluation stage: update of relative transformation matrix, $T_{M}$ and temporary halting of evaluation of the L-system while

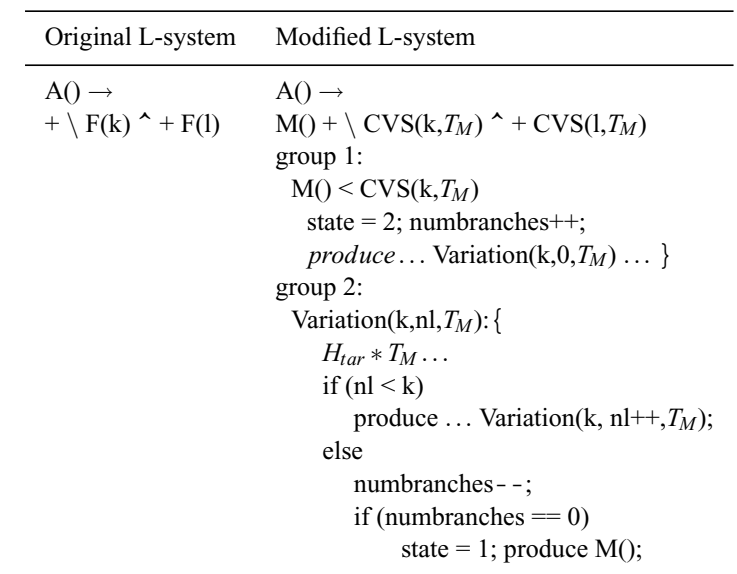

Table 1: Transition from original L-system description to modified L-system creating variation [KL02].

a straight line production becomes a series of small subsegments altering orientation and introducing variation as outlined. As mentioned some branches grow at a particular angle to either gravity or the main stem. This desired growth direction is simulated by either a globally or locally accumulated transformation matrix, $T_{M} . T_{M}$ transforms the desired growth direction to either a global (relative to gravity) or a local (relative to the parent stem) direction. Halting the L-system is required since variation and start location/orientation of one segment is dependent on the previous. Introducing variation sequentially in this manner ensures variation continuity along the length of a branch or stem and preserves discontinuities of the original base model.

L-systems, are inherently non-sequential and thus three mechanisms were used to ensure sequential evaluation: $T a$ ble L-systems, context sensitivity and a semaphore. Table Lsystems associate a state with each rule or production and as evaluation occurs only those rules in the current global state are considered. Altering the state allows us to halt the original L-system and add variation. A marker $M()$ provides a context to be satisfied to ensure evaluation of only one segment of a single branch at a time and also to prevent evaluation of child branches before their parents to which they are attached. The marker is initially placed at the beginning of the main axiom and migrates down the L-system string as evaluation occurs and variation is added. When variation of one segment is complete, the marker is replaced adding a satisfying context to the next segment. Since multiple branches can develop at once, parallel evaluation of branches is permitted. However, using a global state to maintain sequencing of variation causes problems. Thus, a counting semaphore numbranches prevents state changes until all branches have completed variation of their current segment.

Changing a base L-system to introduce variation is fairly generic (see Table 1). Given a set of turtle transformations (i.e $+, \backslash, \wedge)$ and turtle translations (i.e. $F(l)$ ), from a decomposed L-system two productions with different states are added: CVS which Creates a Varied Segment and Variation. As shown, these productions change the state, update the semaphore and add the context $M()$ as outlined above. 